\title{
Pemetrexed and bevacizumab-containing chemotherapy for pleomorphic carcinoma of the lung
}

\author{
TOMOHIRO TAMURA ${ }^{1}$, GEN OHARA ${ }^{1}$, KATSUNORI KAGOHASHI ${ }^{1}$, MIO KAWAGUCHI ${ }^{2}$, \\ KOICHI KURISHIMA ${ }^{3}$ and HIROAKI SATOH ${ }^{1}$ \\ ${ }^{1}$ Division of Respiratory Medicine, Mito Medical Center, University of Tsukuba, Mito, Ibaraki 310-0015; \\ ${ }^{2}$ Division of Respiratory Medicine, Faculty of Medicine, University of Tsukuba, Tsukuba, Ibaraki 305-8575; \\ ${ }^{3}$ Division of Respiratory Medicine, Tsukuba Medical Center, Tsukuba, Ibaraki 305-8558, Japan
}

Received July 10, 2015; Accepted December 11, 2015

DOI: $10.3892 / \mathrm{mco} .2016 .758$

\begin{abstract}
Pleomorphic carcinoma of the lung is a rare, highly malignant subtype of lung cancer, with a more aggressive clinical course compared with other types of non-small-cell lung cancer (NSCLC). Pemetrexed and bevacizumab are currently evaluated as two of the most reliable chemotherapeutic drugs for advanced NSCLC. We herein report a case of a 68- and a 46-year-old man with recurrent and chemo-naïve pleomorphic carcinoma of the lung, respectively, who were treated with a combination of carboplatin, pemetrexed and bevacizumab. The overall survival after the initiation of chemotherapy was 30 and 8 months, respectively. These cases exhibited a relatively long-term survival with chemotherapy. In the absence of definitive clinical trials, which are unlikely to be performed due to the rarity of this tumor, our cases demonstrated the potential utility of pemetrexed- and bevacizumab-containing chemotherapy. Our results also suggested that pemetrexed-containing chemotherapy may be key to the treatment of pleomorphic carcinoma of the lung.
\end{abstract}

\section{Introduction}

Pleomorphic carcinoma of the lung is a rare, highly malignant tumor, accounting for $0.1-0.4 \%$ of primary lung cancer cases (1-3). It has a more aggressive clinical course compared with other types of non-small-cell lung cancer (NSCLC) (4-6), as well as a poorer outcome $(4,7,8)$. We herein report two cases of pleomorphic carcinoma of the lung, who were treated with pemetrexed-containing chemotherapy. Our results demonstrated that pemetrexed-containing chemotherapy may be key to the treatment of advanced cases with this type of lung cancer.

Correspondence to: Professor Hiroaki Satoh, Division of Respiratory Medicine, Mito Medical Center, University of Tsukuba, Miya-machi 3-2-7, Mito, Ibaraki 310-0015, Japan

E-mail: hirosato@md.tsukuba.ac.jp

Key words: pleomorphic carcinoma, lung cancer, pemetrexed, bevacizumab, chemotherapy

\section{Case report}

Case 1. A 68-year-old man was admitted to the hospital with a productive cough. A chest radiograph revealed a tumor $5 \mathrm{~cm}$ in diameter in the right lung. Bioptic specimens, which were obtained transbronchially, were diagnosed as NSCLC. As the patient did not present with hilar/mediastinal lymph node enlargement or distant metastasis, he underwent right upper pulmonary lobectomy. On postoperative pathological examination, the tumor was diagnosed as pleomorphic carcinoma of the lung (Fig. 1). The examination for epidermal growth factor receptor mutations was negative. As there was microscopic involvement of the ipsilateral mediastinal lymph nodes, 4 courses of postoperative chemotherapy containing cisplatin and vinorelbine were administered. Four months after the end of the treatment (10 months after the diagnosis), the disease recurred as two pulmonary nodules $\leq 1 \mathrm{~cm}$ in diameter in the left lung, detected on follow-up chest computed tomography (CT) scan (Fig. 2). The work-up for systemic metastasis was negative. Two courses of chemotherapy, consisting of carboplatin [area under the curve (AUC) $=5$, day 1, q 28 days), pemetrexed $\left(500 \mathrm{mg} / \mathrm{m}^{2}\right.$, day 1 , q28 days) and bevacizumab $(15 \mathrm{mg} / \mathrm{kg}$, day 1, q28 days) was performed, and the post-treatment evaluation CT scan revealed shrinkage of the two pulmonary lesions (Fig. 3). Thereafter, the patient received an additional two courses of chemotherapy. A follow-up CT scan revealed further shrinkage of the pulmonary tumors. The patient received 20 courses of maintenance chemotherapy consisting of pemetrexed $\left(500 \mathrm{mg} / \mathrm{m}^{2}\right.$, day 1 , q28 days) and bevacizumab $(15 \mathrm{mg} / \mathrm{kg}$, day $1, \mathrm{q} 28$ days) without any severe adverse events. No recurrence was found and the patient remained well for 30 months after the initiation of the chemotherapy for recurrence; however, the patient succumbed to brain infarction.

Case 2. A 46-year-old man was admitted to the hospital with anorexia, fever and weight loss. The blood tests revealed a white blood cell count (WBC) of $22,900 / \mu 1$ with $78 \%$ neutrophils, a red blood cell count of $399 \times 10^{4} / \mu 1$, and a platelet count of $43.2 \times 10^{4} / \mu 1$. The patient's hemoglobin concentration was $11.5 \mathrm{~g} / \mathrm{dl}$ and the hematocrit was $34.8 \%$. On the blood chemistry tests, the serum albumin was $2.9 \mathrm{mg} / \mathrm{dl}$, the alkaline phosphatase $348 \mathrm{U} / \mathrm{l}$, the total bilirubin $0.3 \mathrm{mg} / \mathrm{dl}$, and the creatinine 
$0.57 \mathrm{mg} / \mathrm{dl}$. The serum transaminase levels were within normal limits, and blood serology revealed a C-reactive protein (CRP) level of $15.64 \mathrm{mg} / \mathrm{dl}$. The granulocyte/colony-stimulating factor (G-CSF) level was $246 \mathrm{U} / 1$ (normal, $\leq 39 \mathrm{U} / 1$ ). A sizeable mass was identified in the upper lobe of the right lung by chest radiography (Fig. 4). A biopsy specimen obtained by video-assisted thoracoscopic surgery revealed a poorly differentiated lung carcinoma. The patient received 4 courses of chemotherapy with carboplatin ( $\mathrm{AUC}=5$, day 1, q28 days), pemetrexed (500 mg/m² , day 1 , q28 days) and bevacizumab (15 mg/kg, day 1, q28 days), followed by chemoradiotherapy (60 Gy). WBC decreased to within the normal range and fever subsided tentatively for several weeks after each course of chemotherapy. The response was evaluated as 'stable disease'.

Six months after the initiation of the chemotherapy, the patient experienced sudden abdominal pain. An abdominal CT scan revealed free air and ascitic fluid in the pouch of Douglas (Fig. 5). Although the site of perforation was not identified, the patient was diagnosed with acute peritonitis with gastrointestinal perforation and underwent emergency surgery. During the surgical procedure, a large amount of turbid ascites was found. A perforation was discovered inside the jejunum, $\sim 33.0 \mathrm{~cm}$ from Treitz's ligament. An intestinal resection of $\sim 10 \mathrm{~cm}$ was performed. The intraperitoneal cavity was washed with $8,000 \mathrm{ml}$ saline solution and a functional end-to-end anastomosis was performed. After the operation the patient's condition improved gradually. On pathological examination, the resected tumor was diagnosed as metastatic pleomorphic carcinoma of the lung, which was negative for G-CSF immunostaining. This result may be reflective of a possibly heterogeneous tumor population, in which metastatic tumor cells did not produce G-CSF. One month after the surgery, WBC and CRP increased to $>105,100 / \mu 1$ and $39.06 \mathrm{mg} / \mathrm{dl}$, respectively, and the patient succumbed to the disease 8 months after the initiation of the chemotherapy.

\section{Discussion}

Pleomorphic carcinomas are infrequent, comprising 0.1-0.3\% of all lung tumors (1-3). Several studies have reported that pleomorphic carcinomas are associated with a more advanced stage at presentation and a poorer prognosis $(1,5,8,9)$. Recent data indicate that pemetrexed-containing chemotherapy achieves a good response with prolonged survival in advanced conventional NSCLC (10). By contrast, there is a lack of knowledge regarding the role of chemotherapy in the treatment of locally advanced, metastatic, and recurrent pleomorphic carcinoma of the lung.

Bae et al reported that the current strategy of palliative chemotherapy may be largely ineffective and palliative chemotherapy itself is not justifiable (8). The median overall survival (OS) of their 11 patients who did not receive palliative chemotherapy for advanced pulmonary pleomorphic carcinoma was only 2 months, compared with 8 months for the patients who received palliative chemotherapy (8). Hong et al reported the role of palliative chemotherapy for 12 patients with advanced pulmonary pleomorphic carcinoma (11) and demonstrated that the median OS from the day of initiation of first-line chemotherapy was 8 months (11). Of the 12 patients, 3 survived for 1 year and were treated with TKIs as third-line therapy,
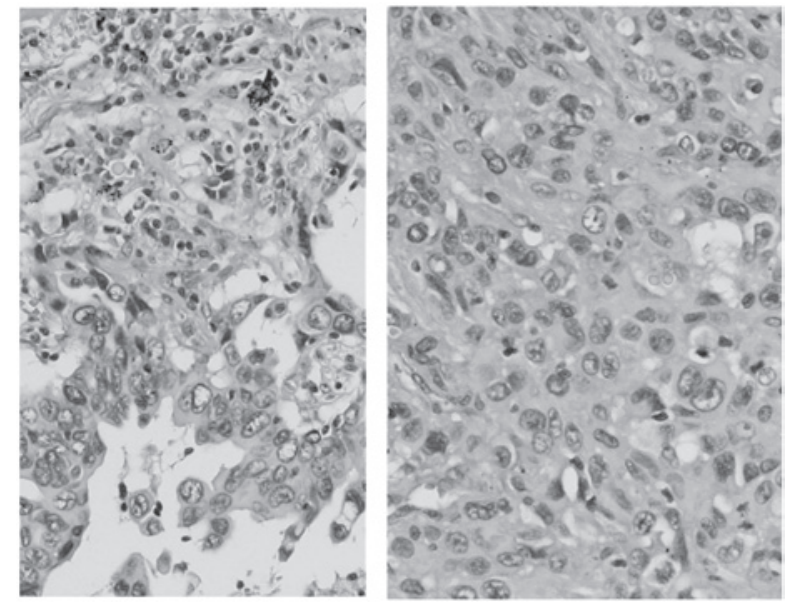

Figure 1. Histological findings of the resected specimens. Giant and spindle cells surrounded by inflammatory cells. Hematoxylin and eosin staining (magnification, $\mathrm{x} 400$ ).

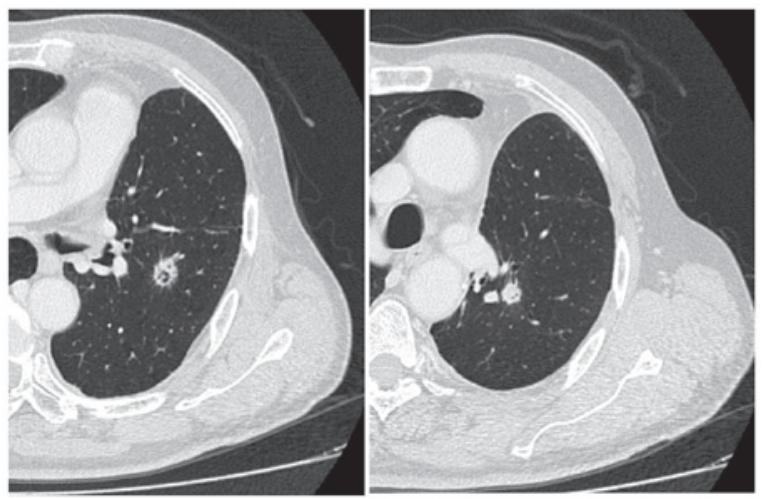

Figure 2. Follow-up chest computed tomography scan shows two pulmonary nodules $\leq 1 \mathrm{~cm}$ in diameter in the left lung.
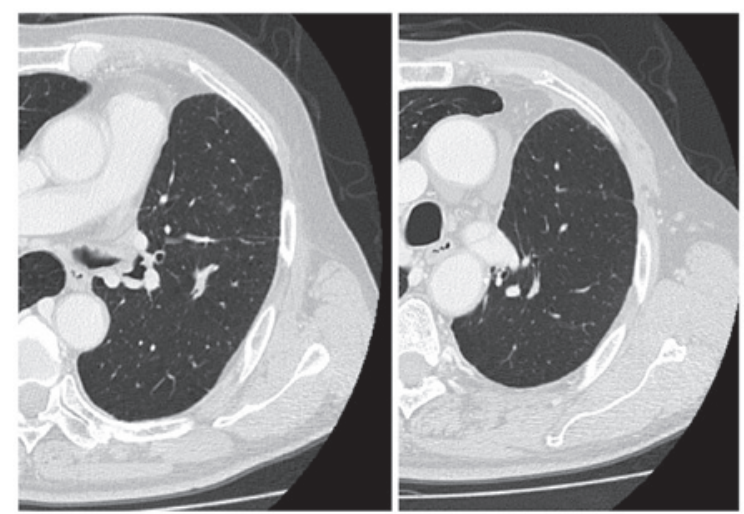

Figure 3. Post-treatment evaluation computed tomography scan shows shrinkage of the two pulmonary tumors.

although the epidermal growth factor receptor (EGFR) mutation status was not described in these patients. Interestingly, 1 of the 3 patients was treated with pemetrexed as second-line chemotherapy (11). In the present study, we demonstrated a sustained response for 30 months in 1 patient and stable disease for 6 months without deterioration of the general condition in another patient treated with pemetrexed-containing 


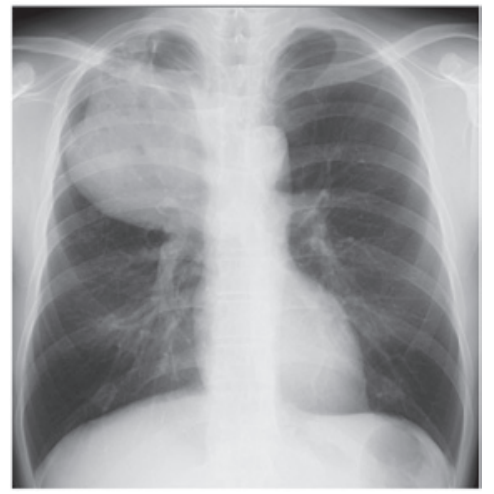

Figure 4. Chest radiograph showing a sizeable tumor mass in the upper lobe of the right lung.

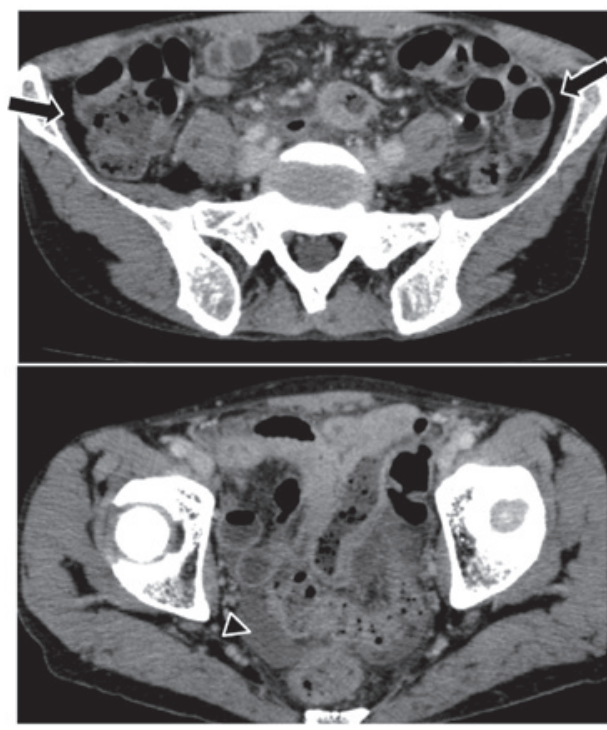

Figure 5. An abdominal computed tomography scan revealed free air (arrows) and ascites in the pouch of Douglas fossa (arrowhead).

chemotherapy. As both patients had wild-type EGFR mutations, they received no TKI therapy. It is well known that the adverse events of pemetrexed are milder compared with those observed with other cytotoxic drugs, such as taxanes and vinorelbine (12). Despite the prolonged administration of pemetrexed and bevacizumab in case 1, there were no severe adverse events $>$ grade 3 , suggesting that cumulative dosing of these drugs was not associated with severe adverse events.

In case 2 , the patient had a G-CSF-producing pleomorphic carcinoma of the lung. To the best of our knowledge, there are no reported treatment results for G-CSF-producing pleomorphic carcinoma of the lung. It is well known that G-CSG-producing tumors tend to have poor prognosis $(13,14)$. The patient was treated with 5 courses of pemetrexed- and bevacizumab-containing chemotherapy. Although the primary tumor exhibited no shrinkage with chemotherapy, prevention of rapid progression and stable disease for 6 months without deterioration of the general condition of the patient was achieved. However, rapid growth of the primary tumor was observed after stable disease, with development of metastases to multiple sites, including the small intestine. We hypothesized that the rapid progression of the disease, including the small intestinal metastasis in our patient, was associated with malignant transformation of the tumor (15). The outcome of the chemotherapy in this case was not satisfactory, although chemotherapy was somewhat effective, without any severe adverse events.

Our cases demonstrated the potential utility of pemetrexedand bevacizumab-containing chemotherapy. Our results also suggest that pemetrexed-containing chemotherapy may be key to the treatment of pleomorphic carcinoma of the lung.

\section{References}

1. Chang YL, Lee YC, Shih JY and Wu CT: Pulmonary pleomorphic (spindle) cell carcinoma: Peculiar clinicopathologic manifestations different from ordinary non-small cell carcinoma. Lung Cancer 34: 91-97, 2001.

2. Ito K, Oizumi S, Fukumoto S, Harada M, Ishida T, Fujita Y, Harada T, Kojima T, Yokouchi H and Nishimura M; Hokkaido Lung Cancer Clinical Study Group: Clinical characteristics of pleomorphic carcinoma of the lung. Lung Cancer 68: 204-210, 2010.

3. Pelosi G, Sonzogni A, De Pas T, Galetta D, Veronesi G, Spaggiari L, Manzotti M, Fumagalli C, Bresaola E, Nappi O, et al: Review article: Pulmonary sarcomatoid carcinomas: A practical overview. Int J Surg Pathol 18: 103-120, 2010.

4. Mochizuki T, Ishii G, Nagai K, Yoshida J, Nishimura M, Mizuno T, Yokose T, Suzuki K and Ochiai A: Pleomorphic carcinoma of the lung: Clinicopathological characteristics of 70 cases. Am J Surg Pathol 32: 1727-1735, 2008.

5. Fishback NF, Travis WD, Moran CA, Guinee DG Jr, McCarthy WF and Koss MN: Pleomorphic (spindle/giant cell) carcinoma of the lung. A clinicopathologic correlation of 78 cases. Cancer 73: 2936-2945, 1994.

6. Rossi G, Cavazza A, Sturn N, Migaldi M, Facciolongo N, Longo L, Maiorana A and Brambilla E: Pulmonary carcinomas with pleomorphic, sarcomatoid, or sarcomatous elements: A clinicopathlogic and immunohistochemical study of 75 cases. Am J Surg Pathol 27: 311-324, 2003.

7. Yamamoto S, Hamatake D, Ueno T, Higuchi T, Hiratsuka M, Shiraishi T, Iwasaki A and Shirakusa T: Clinicopathological investigation of pulmonary pleomorphic carcinoma. Eur J Cardiothorac Surg 32: 873-876, 2007.

8. Bae HM, Min HS, Lee SH, Kim DW, Chung DH, Lee JS, Kim YW and Heo DS: Palliative chemotherapy for pulmonary pleomorphic carcinoma. Lung Cancer 58: 112-115, 2007.

9. Raveglia F, Mezzetti M, Panigalli T, Furia S, Giuliani L, Conforti $S$ and Meda S: Personal experience in surgical management of pulmonary pleomorphic carcinoma. Ann Thorac Surg 78: 1742-1747, 2004.

10. Fuld AD, Dragnev KH and Rigas JR: Pemetrexed in advanced non-small-cell lung cancer. Expert Opin Pharmacother 11: 1387-1402, 2010.

11. Hong JY, Choi MK, Uhm JE, Park MJ, Lee J, Park YH, Ahn JS, Park K, Han JH and Ahn MJ: The role of palliative chemotherapy for advanced pulmonary pleomorphic carcinoma. Med Oncol 26: 287-291, 2009.

12. Chen Y, Wen Q, Liu H, Ao R, Wu X, Guo L, Wang W, He C and Wang J: Pemetrexed versus vinorelbine treatment of advanced non-squamous non-small cell lung cancer in elderly patients. Mol Clin Oncol 1: 553-557, 2013.

13. Shijubo N, Inoue Y, Hirasawa M, Igarashi T, Mori M, Matsuura A, Uede T and Suzuki A: Granulocyte colony-stimulating factor-producing large cell undifferentiated carcinoma of the lung. Intern Med 31: 277-280, 1992.

14. Yamamoto M, Manabe S, Moriyama Y, Ishii H, Tanaka S, Takahashi R, Tomaru K, Kobayashi N, Kudo M, Sasaki M, et al: Long-term remission achieved via combined chemotherapy and radiotherapy in a non-resectable granulocyte colony-stimulating factor producing pleomorphic carcinoma of the lung. Intern Med 52: 2259-2263, 2013.

15. Mao L: Recent advances in the molecular diagnosis of lung cancer. Oncogene 21: 6960-6969, 2002. 